\title{
A challenge to rise to: Importance of the One Health Approach in the Era of the Climate Change Debate
}

\author{
Shyamala Thirunavukkarasu* \\ Department of Molecular Microbiology, Washington University in St Louis, USA
}

*Corresponding author: Shyamala Thirunavukkarasu, Department of Molecular Microbiology, Washington University in St Louis, School of Medicine, 660 S.Euclid Avenue, St Louis 63110, USA

To Cite This Article: Shyamala Thirunavukkarasu. A challenge to rise to: Importance of the One Health Approach in the Era of the Climate Change Debate. Am J Biomed Sci \& Res. 2019 - 5(6). AJBSR.MS.ID.000977. DOI: 10.34297/AJBSR.2019.05.000977.

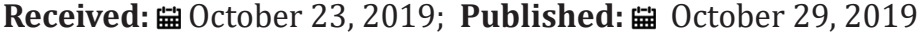

\section{Opinion}

One Health recognizes that the health of people is connected to the health of animals and the environment. It encompasses a collaborative, cross-sectorial, and transdisciplinary approach to achieve the goal of optimal health for humans and animals thriving in a sustainable environment. Statistics indicate that 6 out of every 10 infectious diseases in humans are spread from animals. Recent outbreaks of zoonotic diseases such as SARS and ebola virus infection, as well as the threat of pandemic influenza [1] also serve to highlight the significance of the One Health approach in the infectious disease arena at a global level.

The future of academic research, irrespective of biomedical or epidemiological fields, leans towards interdisciplinary collaboration, which forms the basis of the One Health approach. Interdisciplinary research (IDR) builds bridges between diverse ideas and methodologies thus providing a scope for novel discoveries leading to a competitive edge for securing external research funding. The establishment of dedicated multi/interdisciplinary research buildings in 2019/2020-2021 by several universities such as University of Texas El Paso, Saint Louis University and CalPoly is testament to the acknowledgement of role played by IDR in combating the manifold crisis faced by our world.

While the interdependence of veterinary medicine, human medicine, public health and environmental health can be further illustrated in a variety of ways, nothing is more consequential than global warming in the current world scenario. The collective impact of this inconvenient truth upon human, animal, and environmental health is beginning to unfold at a rate much alarming than previously anticipated.

Global warming apart from affecting the social and environmental determinants of health such as clean air, safe drinking water, nutrition and food security, also impacts food production systems and food safety all of which are closely interlinked. Rising temperatures contribute to habitat destruction which will affect both the animal and human food chains [2,3]. The ecosystems of our mighty oceans are slowly changing. For example, in the arctic ocean the annual production of algae, the base of the food web, was found to increase by 47 percent between 1997 and 2015, resulting in a rapid "greening" of the oceans. The profound impact that this has on animals further up the food chain by determining their metabolism, growth, feeding pattern and survivability is disturbing [4]. It is now estimated that the global fish catch could be reduced 20 percent by 2300 , and by nearly 60 percent across the North Atlantic putting an enormous strain on a prime food source for the global population.

Another critical factor profoundly affected by global climate change is on disease spread and food safety. Humans have known that climatic conditions affect epidemic diseases since the time of the roman aristocrats, who retreated to hill resorts in summer to avoid malaria. Global warming is implicated in the spread of emerging/zoonotic infectious diseases like zika virus, the risk of which is highest at 29C Celsius [5]. Furthermore, Salmonellosis is believed to increase by $5-10 \%$ for each $1^{\circ} \mathrm{C}$ increase in weekly temperature [6]. Thus, as temperatures increase especially in the northern hemisphere it might lead to the spread of these unwelcome disease in areas previously free of such diseases. Also, various changes driven by the indirect effects of climate change influence factors which impact food safety, including antimicrobial resistance and food and water contamination with bacterial and fungal toxins $[7,8]$. Mycotoxins are known to occur more frequently in areas with a hot and humid climate and it is now estimated that $25 \%$ of the world's yearly crop production is contaminated with mycotoxins involving illness or death among particularly vulnerable populations [9]. 
Regardless of the conviction of those with opposing views regarding the impact of climate change/global warming on infectious diseases and public health, it is certain that the epidemiology of infectious diseases will continue to evolve in the years to come. The ability of mankind to react or adapt to this fact depends on the magnitude, speed and willingness of the players on the global arena to embrace change in status quo. The ability for early prediction of epidemics and adoption of preventive strategies necessitates commitment of resources towards research. Targeted research that identifies the host, pathogen, the environment, and society as an interactive system requires interdisciplinary collaboration. Universities and funding agencies are at a pivotal phase in the fight to combat this global phenomenon by encouraging and strengthening the infrastructure to support interdisciplinary research and scientific training.

\section{Conflict of Interest}

The author declares no conflict of interest

\section{References}

1. DE Bloom, D Cadarette (2019) Infectious Disease Threats in the Twenty-First Century: Strengthening the Global Response. Front Immunol 10: 549-549.
2. OL Petchey, PT McPhearson, TM Casey, PJ Morin (1999) Environmental warming alters food-web structure and ecosystem function. Nature 402: 69-72.

3. JP Gibert (2019) Temperature directly and indirectly influences food web structure. Scientific reports 9: 5312 .

4. D Kohlbach, M Graeve, BA Lange, C David, I Peeken, et al. (2016) The importance of ice algae produced carbon in the central Arctic Ocean ecosystem: Food web relationships revealed by lipid and stable isotope analyses. Limnology and Oceanography 61: 2027-2044.

5. H Asad, DO Carpenter (2018) Effects of climate change on the spread of zika virus: a public health threat. Reviews on environmental health 33: 31-42.

6. L Akil, HA Ahmad, RS Reddy (2014) Effects of climate change on Salmonella infections. Foodborne Pathog Dis 11: 974-980.

7. DR MacFadden, SF McGough, D Fisman, M Santillana, JS Brownstein (2018) Antibiotic resistance increases with local temperature. Nature Climate Change 8: 510-514.

8. M Capcarova, K Zbynovska, A Kalafova, J Bulla, P Bielik (2016) Environment contamination by mycotoxins and their occurrence in food and feed: Physiological aspects and economical approach. Journal of environmental science and health 51(4): 236-244.

9. Alshannaq A, Yu JH (2017) Occurrence, Toxicity, and Analysis of Major Mycotoxins in Food. Int J Environ Res Public Health 14(6): 632. 